\title{
A comprehensive study of the effect of in situ annealing at high growth temperature on the morphological and optical properties of self-assembled InAs/GaAs QDs
}

\author{
N. Halder · R. Rashmi - S. Chakrabarti • C.R. Stanley • \\ Miriam Herrera • Nigel D. Browning
}

Received: 27 May 2008 / Accepted: 24 November 2008 / Published online: 13 December 2008

(C) The Author(s) 2008. This article is published with open access at Springerlink.com

\begin{abstract}
We investigate the effect of in situ annealing during growth pause on the morphological and optical properties of self-assembled InAs/GaAs quantum dots (QDs). The islands were grown at different growth rates and having different monolayer coverage. The results were explained on the basis of atomic force microscopy (AFM) and photo-luminescence (PL) measurements. The studies show the occurrence of ripening-like phenomenon, observed in strained semiconductor system. Agglomeration of the selfassembled QDs takes place during dot pause leading to an equilibrium size distribution. The PL properties of the QDs are affected by the Indium desorption from the surface of the QDs during dot pause annealing at high growth temperature $\left(520^{\circ} \mathrm{C}\right)$ subsiding the effect of the narrowing of the dot size distribution with growth pause. The samples having high monolayer coverage (3.4 ML) and grown at a slower growth rate $\left(0.032 \mathrm{ML} \mathrm{s}^{-1}\right)$ manifested two different QD families. Among the islands the smaller are coherent defect-free in nature, whereas the larger dots are plastically relaxed and hence optically inactive. Indium desorption from the island surface during the in situ annealing and
\end{abstract}

N. Halder · R. Rashmi · S. Chakrabarti $(\bowtie)$

Centre of Excellence in Nanoelectronics, Department of Electrical Engineering, Indian Institute of Technology Bombay, Mumbai 400076, Maharashtra, India

e-mail: subho@ee.iitb.ac.in

C.R. Stanley

Department of Electronics \& Electrical Engineering, Rankine

Building, Oakfield Avenue, Glasgow G12 8LT, UK

M. Herrera $\cdot$ N.D. Browning

Department of Chemical Engineering and Materials Science, University of California-Davis, 1153 Kemper Hall, One Shields

Avenue, Davis, CA 95616, USA inhomogeneous morphology as the dots agglomerate during the growth pause, also affects the PL emission from these dot assemblies.

PACS $81.30 \mathrm{Fb} \cdot 68.35 \cdot 78.67 . \mathrm{Hc} \cdot 73.21 . \mathrm{La} \cdot 78.55 . \mathrm{Cr}$

\section{Introduction}

InAs nanostructures embedded in GaAs matrix having a lattice mismatch of about $7.2 \%$, received much attention because of their potential application both for fundamental studies as well as for device applications like QD photodetectors [1, 2], lasers [3, 4], etc. All the applications use either single or stacked layer of QDs in the active region. Hence addressing the issues like controlling the size and coherency, the emission wavelength of the QDs received immense importance, and growth techniques involving Stranski-Krastonov (S-K) growth mode like MOVPE $[5,6]$ and MBE [7, 8] have gained much interest as techniques for producing defect-free InAs/GaAs QDs. Resolution of the above issues necessitates a detailed understanding of the mechanism of nucleation, island transition, etc. in lattice mismatched InAs/GaAs material system by controlling the different growth factors like total amount of InAs material deposited [9, 10], growth temperature [11, 12], growth rate $[12,13]$ and value of $\mathrm{As}_{2}\left(\mathrm{As}_{4}\right)$ pressure or As/In flux ratio $[14,15]$. Another way these issues can be resolved is by developing a suitable post growth scheme like thermal annealing of the active InAs islands just after QD deposition but before encapsulating the dots within the GaAs matrix, i.e. during growth pause [16], which leads to thermallydriven migration or interdiffusion of the material between the QDs. This tailors the size, density and structural properties like coherency of the InAs islands. The change in the 
morphological parameters influences the quantum confinement of the charged carriers in the QDs by modifying their bandgap energy profiles and therefore determines the optical properties and emission wavelength of the heterostructures. In our previous work [17] we have already demonstrated that annealing at high growth temperature during dot pause causes a red shift in the interband photo-response of the self-assembled InAs/GaAs QDs grown by MBE.

In continuation of the above work, in this contribution, we performed further a detailed atomic force Microscopy (AFM) and photo-luminescence (PL) study of the effect of dot pause annealing on InAs/GaAs QDs grown by MBE with different InAs monolayer coverage and at different growth rates. The AFM and PL results were explained considering the influence of In desorption from the island surface and surface diffusion of the In adatoms on the growth plane due to in situ annealing during dot pause.

\section{Growth}

The samples under investigation were grown by solid source MBE in an EPI MOD GEN II system. As shown in Fig. 1, the heterostructure studied here consists of a single layer of InAs QDs of varying InAs coverage being grown on (100) oriented GaAs substrate and encapsulated by GaAs capping layer. For structural characterisation of the grown samples, the heterostructure was terminated with an uncapped InAs QD layer. Reflection high energy electron diffraction (RHEED) was used for in situ monitoring of the growth process as well as the $2 \mathrm{D}$ to $3 \mathrm{D}$ transition.

After native oxide removal from the substrate surface at high temperature, a $0.5 \mu \mathrm{m}$ GaAs buffer layer was grown at $580^{\circ} \mathrm{C}$ over the GaAs substrate. The substrate temperature was then ramped down to $520^{\circ} \mathrm{C}$ along with the growth of $0.1 \mu \mathrm{m}$ GaAs layer over it, to prepare the condition for InAs deposition. Then a variable amount (2.7 ML and 3.4 ML) of InAs material was deposited at $520^{\circ} \mathrm{C}$ to form the InAs QDs

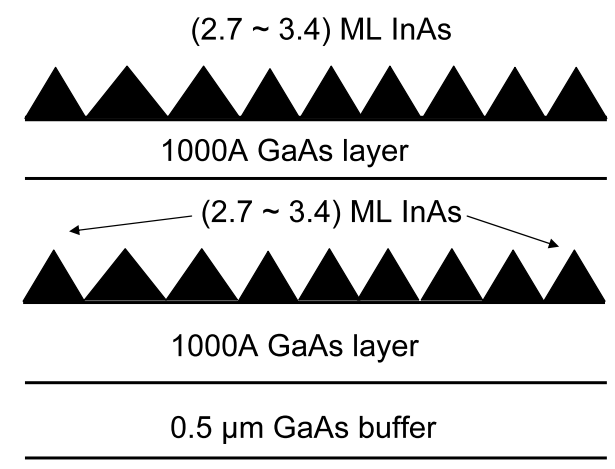

S.I. GaAs substrate

Fig. 1 Schematic diagram of the QD heterostructure sample at two different growth rates $\left(0.032 \mathrm{ML} \mathrm{s}^{-1}\right.$ and $\left.0.2 \mathrm{ML} \mathrm{s}^{-1}\right)$ of Indium. This was followed by in situ annealing of the deposited InAs dots at the growth temperature under an Arsenic pressure of (4-5) $\times 10^{-6}$ torr, at different durations of growth pause ( 0 to $100 \mathrm{~s}$ ). Subsequently, $100 \AA \mathrm{GaAs}$ and $900 \AA \mathrm{GaAs}$ were grown at $520^{\circ} \mathrm{C}$ and $580^{\circ} \mathrm{C}$ respectively in order to cap the dots properly.

The effect of dot pause annealing on the morphology of the grown islands was determined by measuring the parameters like QD diameter and dot density with the help of ex situ AFM. A Veeco Nanoscope-IV system operating in a contact mode with sharpened silicon nitride tip was used for the purpose. To determine the morphology of the QDs in the heterostructure, dark-field scanning transmission electron microscopy (STEM) of the suitably polished and thinned samples was carried out using a JEOL JEM 2500SE electron microscope operated at $200 \mathrm{kV}$. Low temperature (10 K) PL measurements on all samples mounted in a closed cycle $\mathrm{He}-$ lium cryostat were performed using a $\mathrm{He}-\mathrm{Ne}$ laser tuned at $532 \mathrm{~nm}$ at $30 \mathrm{~mW}$ power. The luminescence was dispersed by a $3 / 4 \mathrm{~m}$ grating monochromator and detected by a cooled Ge diode detector using lock-in techniques.

\section{Results}

Figure 2 shows the cross-sectional STEM image of an InAs island from the QD sample grown by MBE at $0.032 \mathrm{ML} \mathrm{s}^{-1}$ and being subjected to $50 \mathrm{~s}$ pause. The STEM picture clearly

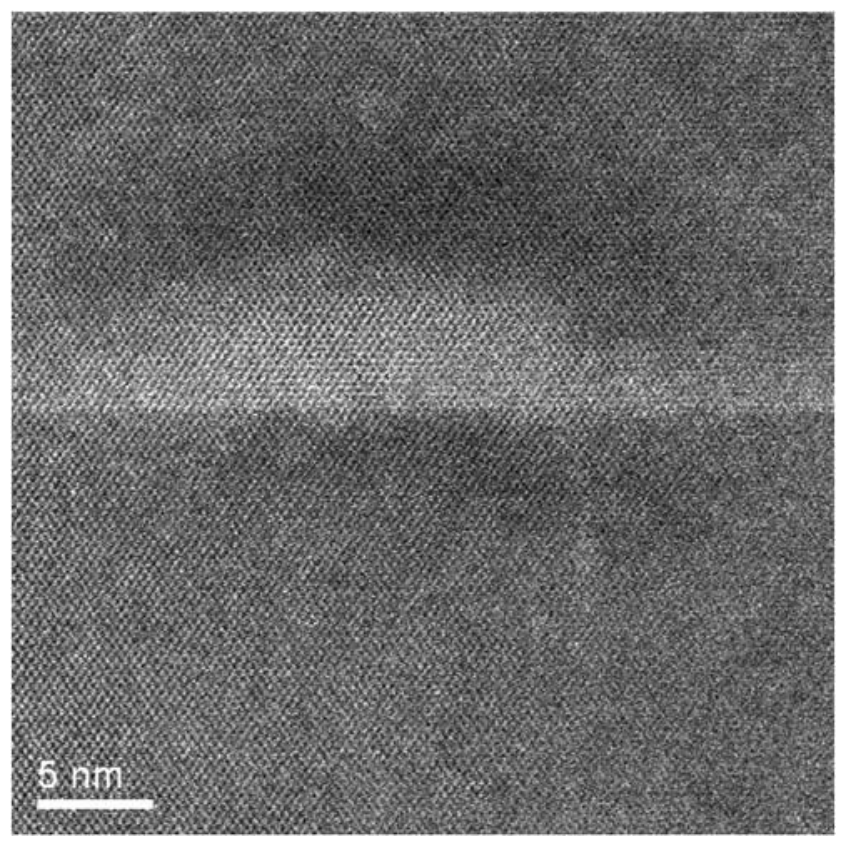

Fig. 2 Cross-sectional dark-field STEM image of the heterostructure sample having 2.7 ML InAs coverage and grown at $0.032 \mathrm{ML} \mathrm{s}^{-1}$ after being subjected to $50 \mathrm{~s}$ of in situ annealing pause 
shows that the island is of pyramidal shape which gives an impression that the self-assembled islands maintain a feasible geometrical shape even after annealing at high growth temperature. From the contrast of the STEM image, the coherent nature of the island can be concluded.

In Figs. 3 and 4 we present the contact mode AFM image of the samples having 2.7 ML InAs coverage being grown at different growth rates and at varied growth pauses, along with the histograms showing the dot size distribution. From the figures it is evident that ripening-like behaviour, observed in most of the heteroepitaxial systems [18, 19], occurs at the time of in situ annealing during dot pause at high growth temperature $\left(520^{\circ} \mathrm{C}\right)$. It is reported that in strained semiconductor systems, the growth pause applied after the formation of self-assembled QDs favours the adatom transfer towards preferential sites on the growth plane like previously formed dots [20]. As the grown islands get sufficient time to agglomerate preferentially during the pause, the dot size distribution shifts to larger values approaching an equilibrium distribution. This phenomenon causes significant changes in the QD density, volume and composition of the QDs, which in turn tailors the emission wavelength from the dots and leads to inhomogeneous broadening of the PL spectrum. In case of the samples grown at a low growth rate of $0.032 \mathrm{ML} \mathrm{s}^{-1}$ and having 2.7 ML InAs monolayer coverage, the initial dot density calculated from the AFM image is $1.91 \times 10^{10} \mathrm{~cm}^{-2}$ which after being subjected to in situ annealing of $50 \mathrm{~s}$ at high growth temperature during growth pause reduces to $1.49 \times 10^{10} \mathrm{~cm}^{-2}$. In the same way for the samples containing 2.7 ML InAs coverage dots grown at $0.2 \mathrm{ML} \mathrm{s}^{-1}$ of Indium, the initial dot density of $5.37 \times 10^{10} \mathrm{~cm}^{-2}$ after similar in situ annealing during $50 \mathrm{~s}$ dot pause reduces to $3.10 \times 10^{10} \mathrm{~cm}^{-2}$.

Here we will establish a connection between the morphological properties of the samples from the ex situ AFM measurements and their emission properties from the PL measurements. From the AFM measurements of Figs. 3 and 4 it is observed that the size distributions of the islands in the as-grown heterostructures, as well as being subjected to annealing during growth pause, contains a narrow distribution of sharp peaks which represents coherent QDs, whose size distribution is controlled by the heteroepitaxial strain in the InAs wetting layer as well as by the surface diffusion of Indium adatoms. In addition to these peaks there are some weak side peaks which represent irregular sized islands. These islands having non-uniform size distribution are relaxed and incoherent in nature due to the presence of dislocations, and the distribution of these irregular sized islands is controlled by a surface diffusion of adatoms at elevated temperatures, not by strain [21]. In Figs. 3 and 4, the histograms show that with the increase in annealing time, the number of side peaks in the size distributions gradually reduces giving a narrow size dispersion and the mean dot size is shifted towards larger values. This phenomenon can be ascribed to the Indium adatom surface diffusion activated at high growth temperature $\left(520^{\circ} \mathrm{C}\right)$.

The PL spectra of the samples having 2.7 ML InAs coverage and grown at different growth rates with varying dot pause are depicted in Figs. 5 and 6. Compared to the dots grown at higher growth rate the PL spectrum of the QD samples grown at a low growth rate of $0.032 \mathrm{ML} \mathrm{s}^{-1}$ is redshifted due to the increased dot size and hence greater quantum confinement for the latter QDs. This is also evident from the AFM images of Figs. 3 and 4. Now comparing the individual PL spectra of Figs. 5 and 6, it can be observed that along with the duration of dot pause the ground state (GS) PL emission for the QD samples was shifted to longer wavelengths and this can only be attributed to the formation of large dots with the in situ annealing due to increased surface diffusion mobility of the In adatoms at the high growth temperature. A small blue shift in the emission, observed in case of the samples grown at $0.2 \mathrm{ML} \mathrm{s}^{-1}$ and being allowed to in situ annealing for $50 \mathrm{~s}$, can be attributed to degradation in dot morphology because of In desorption at high substrate temperature $\left(>520^{\circ} \mathrm{C}\right)$, from low Indium fraction containing islands. It has been reported that QDs grown at a higher growth rate have smaller aspect ratio and have lower Indium content [22] than the islands grown at comparatively slower rate. Further, the high integrated PL intensity in case of the samples which were encapsulated just after the growth pause gives an essence that the dot density is maximal just after the growth pause and with the in situ annealing during the pause agglomeration of the grown islands takes place due to ripening.

The PL line widths for the samples grown at two different growth rates are plotted in Fig. 7. It is observed from the plot that the percentage change in the PL line width with dot annealing time is greater for the dots grown at a slow growth rate than for the dots grown at a faster rate of $0.2 \mathrm{ML} \mathrm{s}^{-1}$. This suggests that the PL emission from the dots grown at slow growth rate and hence having higher Indium content, is considerably affected by the dot pause annealing. Anyway, the reduction in the incoherent island density with in situ annealing time as evident from the AFM images, results in the gradual decrease in the FWHM of the GS emission peak from the dots plotted in Fig. 7, only up to $25 \mathrm{~s}$ of dot pause annealing, in case of the dots grown at lower growth rate. But after being allowed to in situ anneal for $50 \mathrm{~s}$ the FWHM of GS PL emission from these dots increases considerably, although there is a reduction in the size dispersion of the dots with annealing time. This degradation in the optical properties of the dots grown at $0.032 \mathrm{ML} \mathrm{s}^{-1}$ after $50 \mathrm{~s}$ of growth pause is due to Indium atom desorption from the surface of the dots which degrades the homogeneity in the composition of the self-assembled islands and also results in the reduction of effective QD volume [23]. As the QDs grown at 

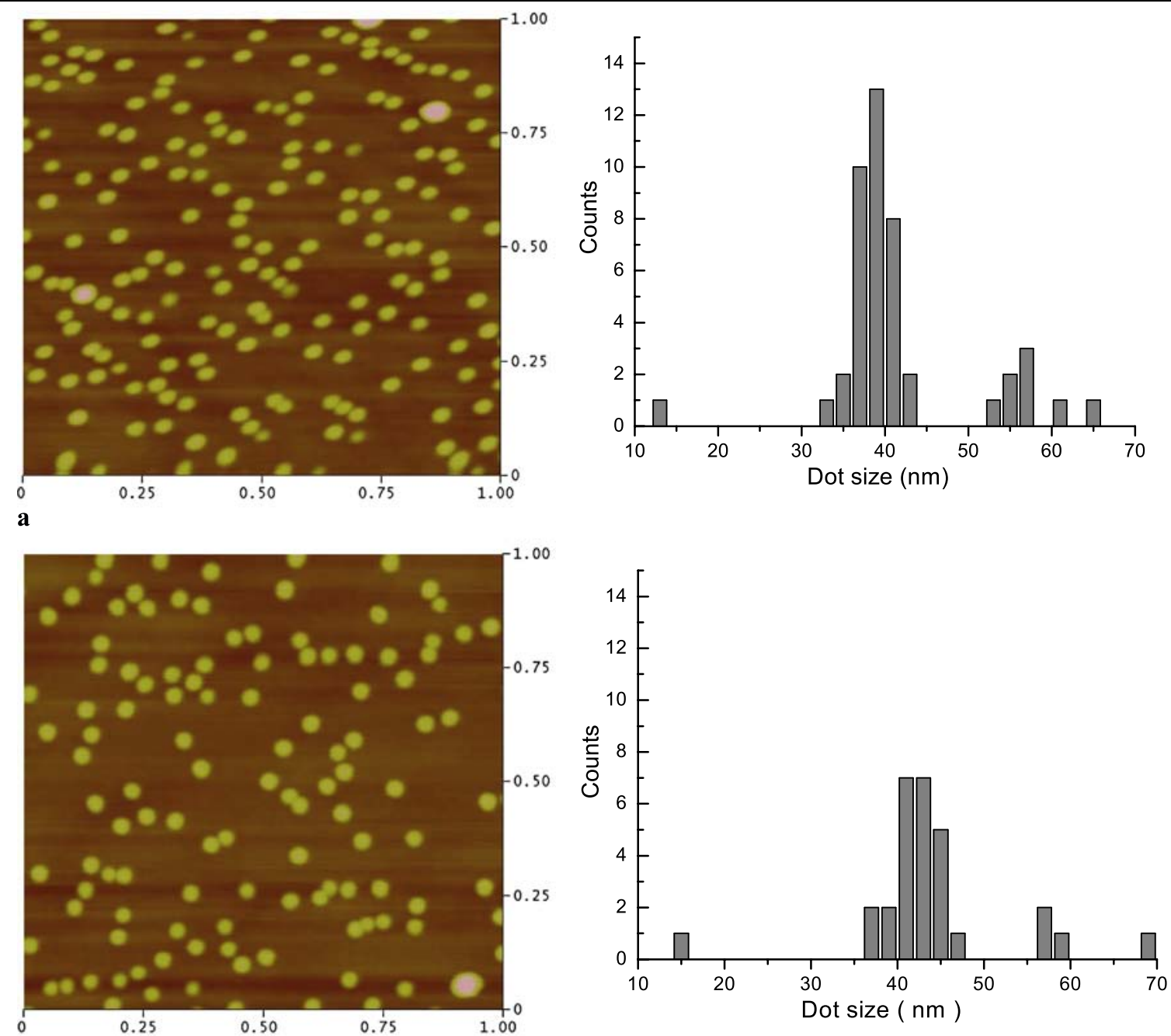

b
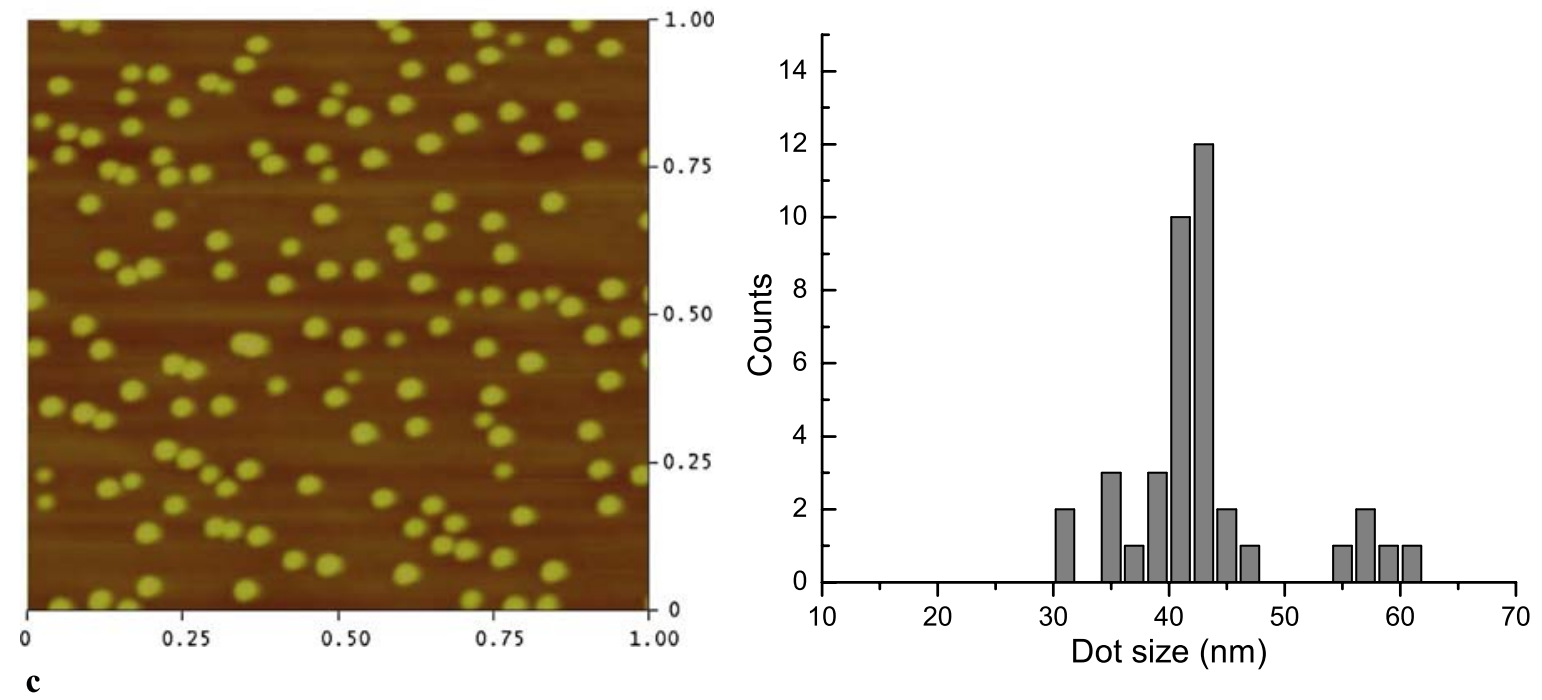

Fig. 3 AFM $(1 \times 1 \mu \mathrm{m})$ top views of the InAs/GaAs QDs having 2.7 ML InAs coverage grown at $0.032 \mathrm{ML} \mathrm{s}^{-1}$ after a $0 \mathrm{~s} \mathrm{~b} 25 \mathrm{~s} \mathrm{~d} 50 \mathrm{~s}$ of growth pause at $520^{\circ} \mathrm{C}$. The size histograms associated to each image are shown on the right 



Fig. 4 AFM $(1 \times 1 \mu \mathrm{m})$ top views of the InAs/GaAs QDs having 2.7 ML InAs coverage grown at $0.2 \mathrm{ML} \mathrm{s}^{-1}$ after a $0 \mathrm{~s} \mathbf{b} 25 \mathrm{~s} \mathbf{d} 50 \mathrm{~s}$ of growth pause at $520^{\circ} \mathrm{C}$. The size histograms associated to each image are shown on the right 


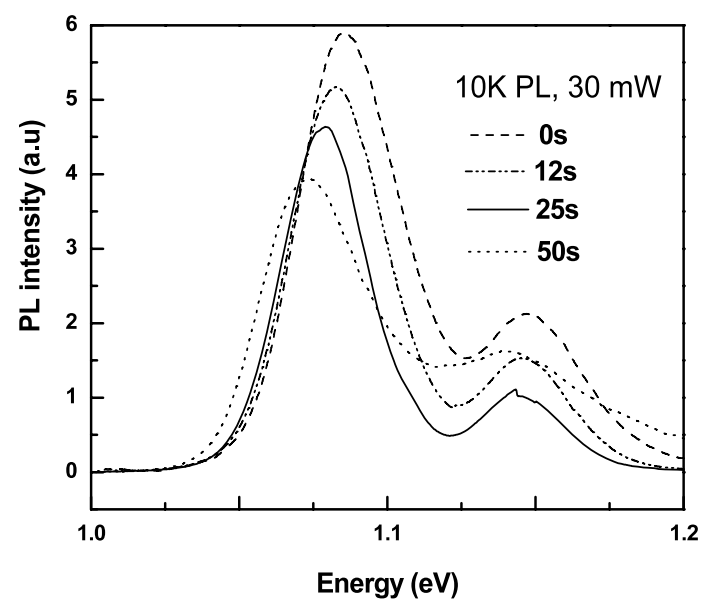

Fig. 5 PL spectra for the InAs QD samples having 2.7 ML InAs coverage grown at $0.032 \mathrm{ML} \mathrm{s}^{-1}$ with $0 \mathrm{~s}, 12 \mathrm{~s}, 25 \mathrm{~s}$ and $50 \mathrm{~s}$ ripening pause

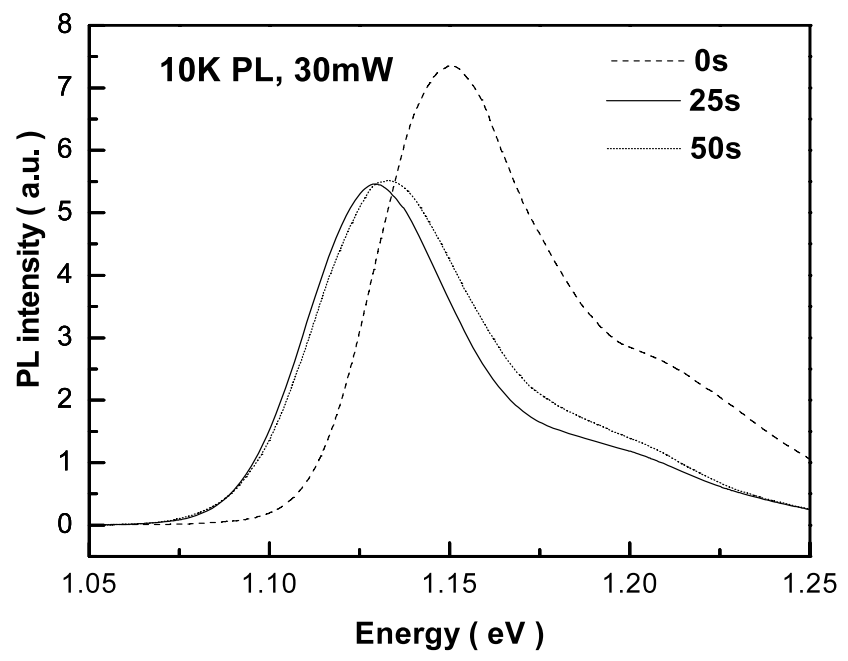

Fig. 6 PL spectra for the InAs QD samples having 2.7 ML InAs coverage grown at $0.2 \mathrm{ML} \mathrm{s}^{-1}$ with $0 \mathrm{~s}, 25 \mathrm{~s}$ and $50 \mathrm{~s}$ ripening pause

higher growth rate have lower indium content compared to the islands being grown at slower rate, the effect of Indium desorption from the island surface at higher growth temperature, which takes place in competition with the adatom diffusion during growth pause, is more pronounced in case of the former QDs. This leads to increase in FWHM of the GS emission from these dots with the growth pause, subsiding the effect of the narrowing of the dot size distribution with in situ annealing. After $50 \mathrm{~s}$ of annealing pause the morphological properties of the dots degrade drastically leading to a blue shift in the PL spectra, as mentioned earlier. Some of the above results shown here have already been reported earlier [17], but for the sake of better comprehensiveness and clarity, also have been included in this paper.

For an in-depth study of the effect of monolayer coverage on the ripening phenomenon, we performed AFM and

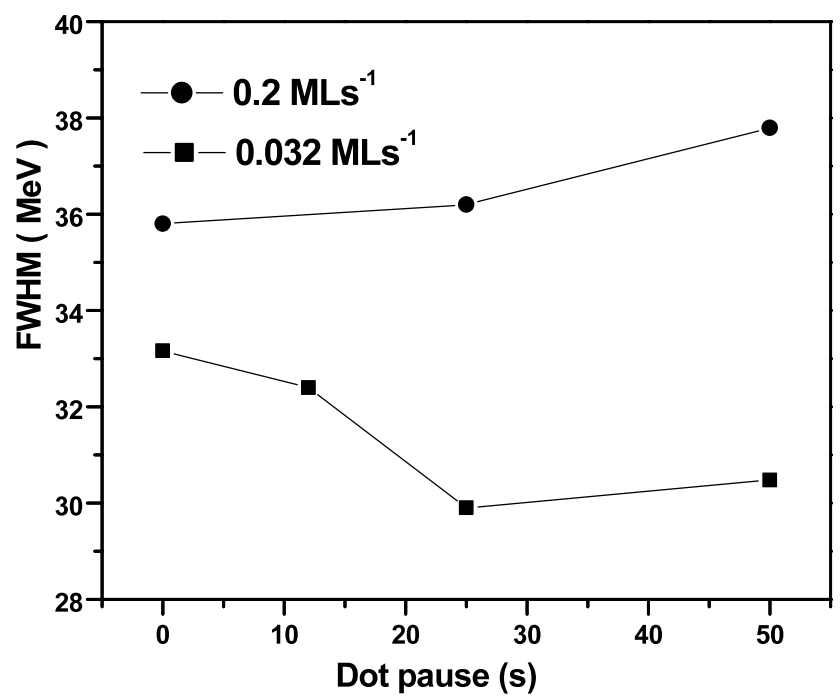

Fig. 7 FWHM of the ground state PL emission peak of the QD samples having 2.7 ML InAs coverage grown at $0.032 \mathrm{ML} \mathrm{s}^{-1}$ and $0.2 \mathrm{ML} \mathrm{s}^{-1}$, plotted as a function of the duration of dot pause

PL studies on the QD samples grown at $0.032 \mathrm{ML} \mathrm{s}^{-1}$ having 3.4 ML of InAs coverage and subjected to in situ annealing at the high growth temperature. The AFM images for the samples in Fig. 8, subjected to dot pause varying from 25 to $100 \mathrm{~s}$, clearly show that the QD samples contain two differently sized dot families. The high density smaller dots of height $6 \sim 8 \mathrm{~nm}$ have uniform size distribution, whereas the large irregular dots are of height $13-15 \mathrm{~nm}$. In self-assembled growth, for increased monolayer coverage at slow deposition rate there is little increase in the density of small regular islands [13]. But there is a considerable increase in the density of large irregular shaped 3D islands which are plastically relaxed due to the accumulation of strain related defects. These large islands are the favourable sites for the attachment of adatoms during in situ annealing [13]. The adatoms migrate across the growth surface and clusters at the large islands as a result of the enhancement of the surface diffusion mobility of the adatoms during annealing at elevated temperature. Thus it is evident from the AFM images that with the increase in in situ annealing time of the samples at the $520^{\circ} \mathrm{C}$, the smaller QD density decreases, but the density of the larger dots increases. Figure $8 \mathrm{c}$ of the samples subjected to $100 \mathrm{~s}$ growth pause shows an increase in the QD height fluctuation, where few of the large islands have ripened to $28 \mathrm{~nm}$ height. The optical data for the samples shown in Fig. 9 follow a trend correlated with the AFM data. The PL spectra can be explained easily by taking into account that the smaller islands are elastically relaxed dislocation-free, whereas the large irregular shaped islands are plastically relaxed incoherent and hence optically inactive. Therefore the PL spectra of Fig. 9 actually represent the smaller QDs. Figure 9 shows that the spectra contain three peaks unlike those of Figs. 5 and 6, and the third peak 

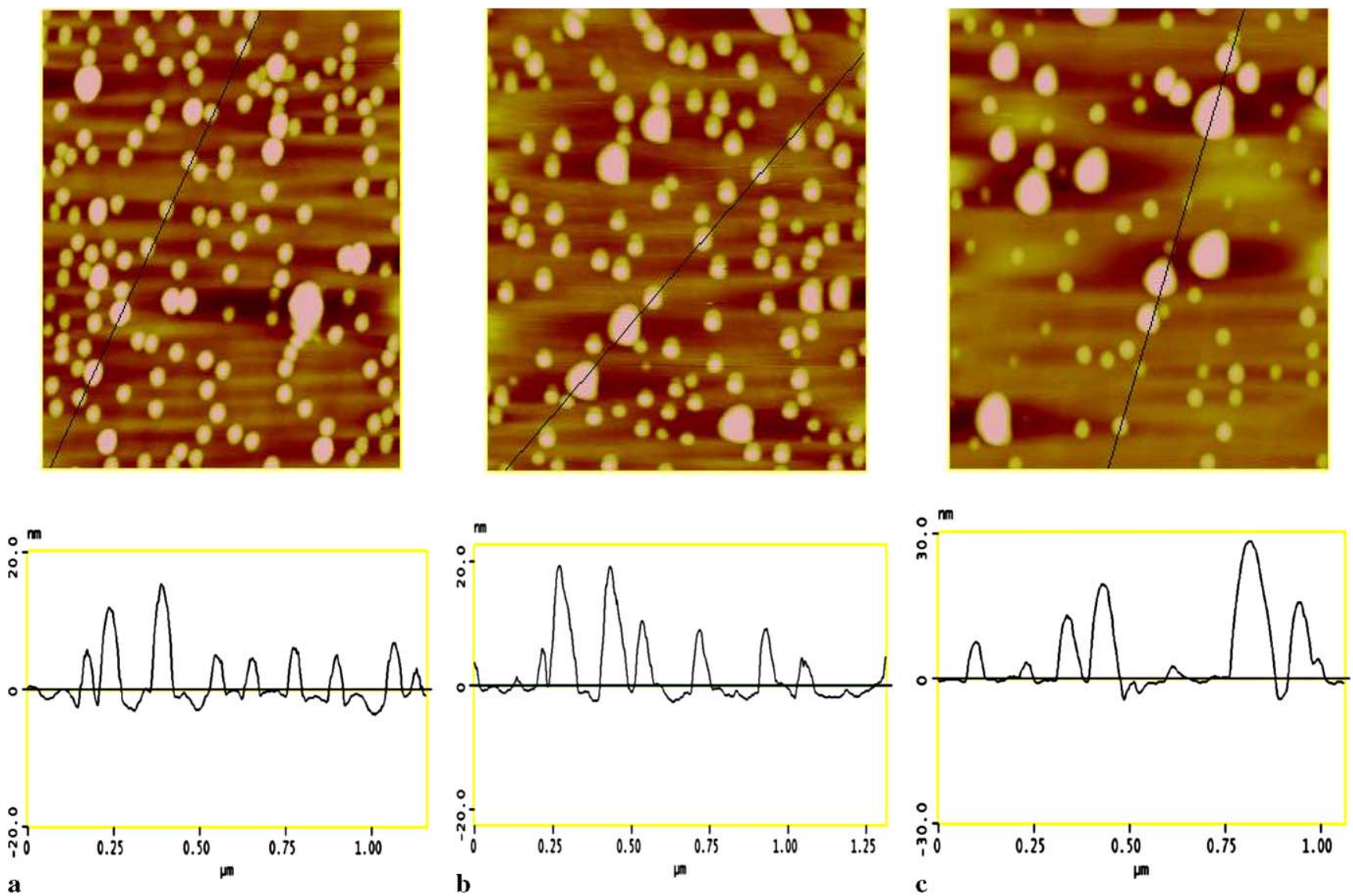

Fig. 8 AFM $(1 \times 1 \mu \mathrm{m})$ top views of the InAs/GaAs QDs having $3.4 \mathrm{ML}$ InAs coverage grown at $0.2 \mathrm{ML} \mathrm{s}^{-1}$ after $\mathbf{a} 25 \mathrm{~s} \mathbf{b} 50 \mathrm{~s} \mathrm{~d} 100 \mathrm{~s}$ of growth pause at $520^{\circ} \mathrm{C}$. The line profiles of the islands presented in the respective QD samples are given below



Fig. 9 PL spectra for the InAs QD samples having 3.4 ML InAs coverage grown at $0.032 \mathrm{ML} \mathrm{s}^{-1}$ with $25 \mathrm{~s}, 50 \mathrm{~s}$ and $100 \mathrm{~s}$ ripening pause

is due to the second excited state of large-sized QDs grown at greater InAs monolayer coverage [24].

Now it is important to note that with the increase in in situ annealing just after the island deposition is stopped, the peak representing the GS transition in the PL spectrum of the QD samples in Fig. 9 and consequently the other peaks, are blue-shifted. For $25 \mathrm{~s}$ annealing during the dot pause the GS peak in the spectrum is at $1.07 \mathrm{eV}$, whereas the same is at $1.11 \mathrm{eV}$ for $100 \mathrm{~s}$ pause. This blue shift in the PL emission spectra can only be related to the Indium desorption from the island surface due to increased annealing time at high growth temperature during dot pause, resulting in the inhomogeneity in QD composition and height distribution. Further, the dot density also decreases with dot pause, as depicted in Fig. 10. The FWHM broadening of the GS transition for the QD samples having 3.4 ML of InAs coverage and grown at the reduced growth rate $\left(0.032 \mathrm{ML} \mathrm{s}^{-1}\right)$ with dot pause described in Fig. 10, is again attributed to inhomogeneous dot size and height distribution, as well as reduction in homogeneity of the island composition during in situ annealing. The observation of the monotonic decrease in integrated PL intensity with dot pause in the PL spectra of Fig. 9 suggests that due to Indium desorption during growth pause, plastic relaxation through the generation of planer defects occurs in the dots. Also the decrease in the dot density due to agglomeration of islands during growth pause might have 


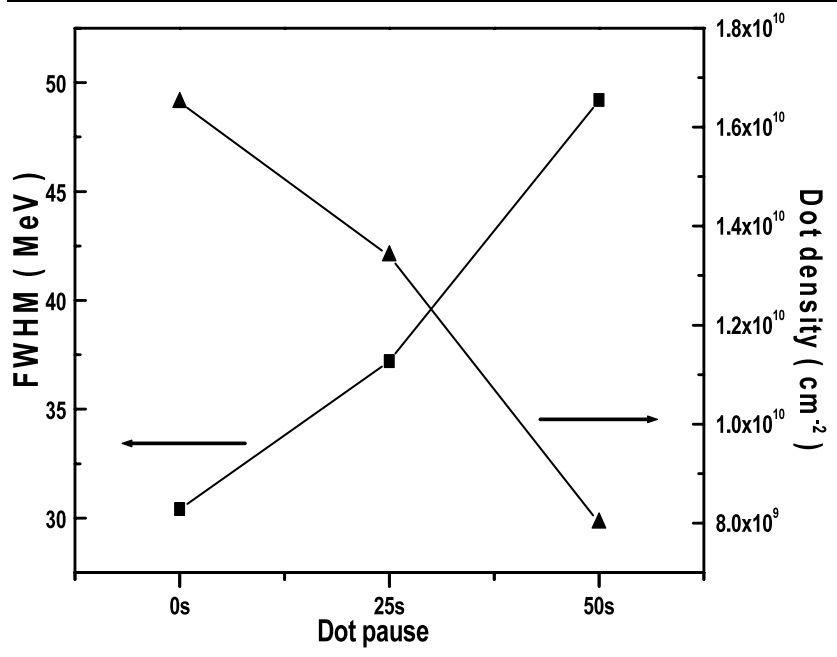

Fig. 10 FWHM of the ground state PL emission peak and dot density for the QD samples having 3.4 ML InAs coverage and grown at $0.032 \mathrm{ML} \mathrm{s}^{-1}$, plotted as a function of the duration of dot pause

some contribution to this PL-integrated intensity reduction with the annealing pause.

\section{Conclusion}

In summary, we have studied the effect of annealing at high growth temperature on the morphological and optical properties of the self-assembled InAs/GaAs QDs during growth pause. We show that agglomeration of the self-assembled islands occurs during annealing and the dots grow in size approaching an equilibrium size distribution. Our results also suggest that the Indium desorption from the surface of the grown QDs during dot pause annealing affects the PL properties of the QDs despite that there is a narrowing of the dot size distribution with growth pause. For the QD samples having high monolayer coverage (3.4 ML) and grown at a slower growth rate $\left(0.032 \mathrm{ML} \mathrm{s}^{-1}\right)$ two differently sized QD families are observed. The smaller dots are defect-free and participate in PL emission, whereas the larger dots are optically inactive due to the accumulation of dislocations. The PL emission from these dots is also affected by Indium desorption from the island surface during the in situ annealing and inhomogeneous dot size and height distribution as a result of agglomeration of dots during the growth pause. So it is preferable to have QDs grown with a lower InAs monolayer coverage, to harness the advantages of higher integrated PL intensity as well as to escape the problems arising from bimodal QD size distribution.
Acknowledgements S.C. has grown the samples while at the University of Glasgow, UK. The authors would like to acknowledge the FIST (Physics)-IRCC Central SPM Facility of IIT-Bombay for the AFM images of the samples. SC acknowledges the support provided by IIT Bombay via seed grant ID. Spons/EE/SCB-1/2007 and also the support of Department of Science and Technology, India, are acknowledged.

Open Access This article is distributed under the terms of the Creative Commons Attribution Noncommercial License which permits any noncommercial use, distribution, and reproduction in any medium, provided the original author(s) and source are credited.

\section{References}

1. A.D. Stiff, S. Krishna, P. Bhattacharya, S. Kennerly, Appl. Phys. Lett. 79, 421 (2001)

2. B. Kochman, A.D. Stiff-Roberts, S. Chakrabarti, J.D. Phillips, S. Krishna, J. Singh, P. Bhattacharya, IEEE J. Quantum Electron. 39, 459 (2003)

3. C. Platz, C. Paranthoen, P. Caroff, N. Bertru, C. Labbe, J. Even, O. Dehaese, H. Folliot, A. Le Corre, S. Loualiche, G. Moreau, J.C. Simon, A. Ramdane, Semicond. Sci. Technol. 20, 459 (2005)

4. Q. Cao, S.F. Yoon, C.Y. Liu, C.Y. Ngo, Nanoscale Res. Lett. 2, 303 (2007)

5. V. Krapek, K. Kuldova, J. Oswald, A. Hospodkova, E. Hulicius, J. Humlicek, Appl. Phys. Lett. 89, 153108 (2006)

6. D. Franke, M. Moehrle, J. Boettcher, P. Harde, A. Sigmund, H. Kuenzel, Appl. Phys. Lett. 91, 081117 (2007)

7. J. Marquez, L. Geelhar, K. Jacobi, Appl. Phys. Lett. 78, 2309 (2001)

8. H. Saito, K. Nishi, S. Sugou, Appl. Phys. Lett. 78, 267 (2001)

9. M.J. da Silva, A.J. Quiry, P.P. Gonzalez-Borrero, E. Marega Jr., J. Cryst. Growth 236, 41 (2002)

10. G.S. Solomon, J.A. Trezza, J.S. Harris Jr., Appl. Phys. Lett. 66, $991(1995)$

11. H. Saito, K. Nishi, S. Sugou, Appl. Phys. Lett. 74, 1224 (1999)

12. O. Suekane, S. Hasegawa, M. Takata, T. Okui, H. Nakashima, Mater. Sci. Eng. B 88, 158 (2002)

13. P.B. Joyce, T.J. Krzyzewski, G.R. Bell, T.S. Jones, E.C. Le Ru, R. Murray, Phys. Rev. B 64, 235317 (2001)

14. G.S. Solomon, J.A. Trezza, J.S. Harris Jr., Appl. Phys. Lett. 66, 3161 (1995)

15. K. Yamaguchi, K. Yujobo, T. Kaizu, Jpn. J. Appl. Phys. Part 239 , L1245 (2000)

16. H.W. Ren, K. Nishi, S. Sugou, M. Sugisaki, Y. Masumoto, Jpn. J. Appl. Phys. Part 1 36, 4118 (1997)

17. N. Halder, S. Chakrabarti, C.R. Stanley, J. Nanosci, Nanotechnol. (2008, in press)

18. K. Poetschke, L. Mueller-Kirsch, R. Heitz, R.L. Sellin, U.W. Pohl, D. Bimberg, N. Zakharov, P. Werner, Physica E 21, 606 (2004)

19. T.J. Krzyzewski, T.S. Jones, J. Appl. Phys. 96, 668 (2004)

20. T.T. Ngo, P.M. Petroff, H. Sakaki, J.L. Merz, Phys. Rev. B 53, 9618 (1996)

21. T. Suzuki, Y. Temko, K. Jacobi, Phys. Rev. B 67, 045315 (2003)

22. P.B. Joyce, T.J. Kizyzewski, G.R. Bell, T.S. Jones, S. Malik, D. Child, R. Murray, Phys. Rev. B 62, 10891 (2000)

23. H. Lee, R.R. Lowe-Webb, W. Yang, P.C. Sercel, Appl. Phys. Lett. 71, 2325 (1997)

24. M. Grundmann, N.N. Ledentsov, O. Stier, D. Bimberg, V.M. Ustinov, P.S. Kopev, Zh.I. Alferov, Appl. Phys. Lett. 68, 979 (1996) 